\title{
Half a Century of Care
}

\author{
Mary Ann McColl \\ Queens University, Canada
}

\begin{abstract}
$T$ he issue of long-term care and support of people with acquired brain injury has been a significant clinical and policy issue for some time; however. as evidence accrues about increases in incidence and survival from brain injury, the focus on this issue sharpens (Kolpan, 1990; McCluskey, 2005). The net effect of these increases (in the number of new brain injuries per year plus survival beyond the acute phase) is a sharp increase in prevalence, or the number of people living with the effects of brain injury in a given population. Add to that new information that has recently been published regarding long-term survival, and it becomes immediately apparent that factors are converging to produce an immanent increase in the economic and human costs of acquired brain injuries, at least in the developed world.
\end{abstract}

Although relatively little information is available on long-term survival following brain injury, recent studies based on large samples (e.g., the American Model Systems dataset) suggest that there is only a small decrease in life expectancy among those who survive the first year after brain injury - in the range of 3 to 7 years (Brown, Leibson, Malec, Perkins, Diehl et al., 2004; Harrison-Felix, Whiteneck, DeVivo, Hammond \& Jha, 2004; Shavelle, Strauss, Whyte, Day \& Yu, 2001). While age and gender-specific mortality is somewhat elevated relative to the nondisabled population, much of this risk is accounted for by several specific causes of death - seizurerelated deaths, choking and aspiration, and suicide. Those at greatest risk are those with severe mobility restrictions. Those who were ambulatory had life expectancies closer to general population values. Thus for a person injured in his or her twenties, it is not inconceivable that he or she might require half a century of care.

The most recent published study by Lannoo, Brusselmans, VanEynde, VanLaere and Stevens (2004) estimated the prevalence of brain injury at 183 per 100,000 (Belgium). This estimate is compatible with established British estimates, which find prevalence between 100 and 150 per 1000 (Bryden, 1989). Therefore, in a medium-sized city like the one in which I live (Kingston, Ontario, Canada; population 120,000), there must be about 200 people living with a brain injury. Of those, according to Lannoo, about half, or 100, need consistent lifelong support to be able to continue to live in the community and function independently. A further $20 \%$ (or 40 people) need a level of care that requires institutionalisation. A fortunate $20 \%$ (40) will be involved in educational or other productive activity during the day, but half (100 people) will find the lack of productivity a huge problem.

Mellick, Gerhart and Whiteneck (2003) have described six typical pathways that individuals follow in the postacute phase after brain injury. A surprising $64.5 \%$ leave acute care and receive no further services. Two of the pathways include long-term care (either with or without rehabilitation prior), representing about $6 \%$ of postacute patients. Although these tend to be older, more challenging clients, there is also a suggestion that economic factors and institutional environments contribute to significantly poorer short and long-term outcomes than the various community discharge destinations. At the present time, those who wind up as long-term care residents tend to be male with an average age of 53, and with severe cognitive and/or physical disabilities (Buchanan, 2003). According to O'Reilly (2002), the population with acquired brain injuries in nursing homes is expected to grow, and this is an entirely unsatisfactory response to the growing need for long-term care.

O'Keefe (1994) reports, and anyone who works in the system knows, that long-term care 
and support of people with acquired brain injuries is a patchwork of goods and services - some government funded, some privately funded (insurance or out-of-pocket), some charitable, and some voluntary (usually from family or friends). The ability to take advantage of the available resources is highly idiosyncratic - as Degeneffe (2001) puts it, based on the demographic and socioeconomic characteristics of the individual and his or her immediate supports. And yet, the availability of durable supports has repeatedly been shown to be one of the most deterministic factors in successful community integration and positive health outcomes. Tomberg, Toomela, Pulver and Tikk (2005) go so far as to suggest that the development of support resources should be the main focus of rehabilitation, even over functional skill development, due to its highly predictive role in postacute outcomes.

Social support has traditionally been regarded as a buffer against stress of virtually any type (McColl, 1995; McColl \& Skinner, 1988). Almost every human condition - from bereavement to pregnancy, from unemployment to workplace stress, from natural disasters to posttraumatic stress - has been shown to benefit from the mediating power of social support. It is an integral part of our conception of community integration (McColl, Carlson, Johnston, Minnes, Shue et al., 1998; McColl, Davies, Carlson, Johnston, \& Minnes, 2001; Minnes, Carlson, McColl, Nolte, Johnston et al., 2003). Along with having somewhere suitable to live and something worthwhile to do with one's time, it is an essential component of the ability to find a place for oneself in the community.

Social support may be defined in a number of ways: according to the number of social contacts a person has in a given period of time (objective quantitative approach); as the extent to which individuals perceive that support is both available and adequate; in terms of the types of support provided - instrumental, emotional or informational. Social support may be considered by source - intimate relationship, confidante, family, friends, neighbours, work associates, casual acquaintances. It may be differentiated as formal (a relationship to which anyone can have access by virtue of a certain eligibility criteria) or informal (a relationship that is only available to the individual because of who he or she is). Finally support may be considered on the basis of an evaluation of satisfaction. I have even wondered, despite all the literature that has been generated regarding the measurement of social support, if the issue might be reducible to the single question, 'Is there someone that you can count on should the need arise?'

Such is the robust nature of the relationship between social support and health outcomes that virtually every way of measuring support has been associated with positive findings. So compelling is the literature on social support that it has been said to be even more important than smoking as a single determinant of health. But the literature is equivocal on whether social support works the same way for people with brain injuries. On one hand, both qualitative and quantitative studies have found that social support among adults with brain injuries was indeed related to quality of life outcomes (Petrella, McColl, Krupa, \& Johnston, 2005; Tomberg et al., 2005). On the other hand, Rutterford and Wood (2006) suggest that there are so many other powerful mediators of the relationship between support and stress for someone with a brain injury (such as functional and psychosocial variables), that the stress adjustment model simply may not work.

When talking about social support for someone with a brain injury, it is probably important to differentiate between three terms that are often used interchangeably - support, care and supervision. It is also important to specify the degree of responsibility associated with the support relationship. One of the most contentious issues regarding social support in our research and that of others has been the issue of risk tolerance, and the necessity to calibrate the degree of independence against the degree of risk (McColl, Davies, Carlson, Johnston, Harrick et al., 1999). As Petrella, McColl, Krupa and Johnston (2005) point out, space for self-discovery, recognition of vulnerability, and the opportunity for experimentation were essential elements of successful community integration, and none of these can be achieved without a degree of tolerance for potential risks.

In closing, I am tempted to reflect from a personal perspective on the issue of long-term planning for personal and financial security. As many of us from the baby-boom generation contemplate the prospect of retirement, aging and eventual long-term care, we undertake a process that carries with it some degree of foreboding about what the future holds. We are forced to confront questions of a 'What if ...?' nature - questions that take us into the fearful territory of the unknowable. Imagine then the layers of complexity that are heaped on this process for someone who is planning for a 50 -year time window rather than a 20 -year one; for someone whose needs include the broad range of services often required by someone with a brain injury; for someone who knows that 
many of the informal supports on whom he or she depended for the bulk of his or her life are unlikely to still be available.

Articles in this special issue of Brain Impairment address many of the issues covered in the current editorial. On the substantive side, authors have addressed the importance of meaningful participation, risk tolerance and social support. They have explored alternatives to institutional residential care, and they have focused on particularly challenging subsets of the population, such as people with dual diagnoses or persistent behavioural problems. On the methodological side, these articles cover policy analysis, research synthesis, qualitative studies, small sample, descriptive and survey research. They illustrate the potential for a variety of different approaches to contribute to an enhanced understanding of this complex issue. I look forward to sharing with the readers the privilege of reading the selection of articles assembled for this special issue, and to learning more from this august group of authors.

\section{References}

Brown, A.W., Leibson, C.L., Malec, J.F., Perkins, P.K., Diehl, N.N., \& Larson, D.R. (2004). Long term survival after traumatic brain injury: A populationbased analysis. NeuroRehabilitation, 19(1), 37-43.

Bryden, J. (1989). How many injured. The epidemiology of post-head injury disability. In R. Wood \& P. Eames (Eds.), Models of brain injury rehabilitation. London: Chapman \& Hall.

Buchanan, R.J., Wang, S., \& Huang, C. (2003). Profiles of nursing home residents with traumatic brain injury using the Minimum Data Set. Brain Injury, 17(6), 507-523

Degeneffe, C.E. (2001). Family caregiving and traumatic brain injury. Health \& Social Work, 26(4), 257-268.

Harrison-Felix, C., Whiteneck, G., DeVivo, M., Hammond, F.M., \& Jha, A. (2004). Mortality following rehabilitation in the traumatic brain injury Model Systems of Care. NeuroRehabilitation, 19 (1), 45-54.

Kolpan, K.I. (1990). Medicolegal issues regarding lifelong care. Journal of Head Trauma Rehabilitation, 5(1), 100-101.

Lannoo, E., Brusselmans, W., VanEynde, L., VanLaere, M., \& Stevens, J. (2004). Epidemiology of acquired brain injury in adults: Prevalence of long-term disabilities and resulting needs for ongoing care in the region of Flanders Belgium. Brain Injury, 18(2), 203-211.

McCluskey, M.A. (2005). A grounded theory of care management after traumatic brain injury [Thesis abstract]. Australian Occupational Therapy Journal, 52(1), 93-94.

McColl, M.A., Davies, D., Carlson, P., Johnston, J., \& Minnes, P. (2001). The Community Integration Measure: Development and preliminary validation. Archives of Physical Medicine and Rehabilitation, $82,429-434$.

McColl, M.A., Davies, D., Carlson, P., Johnston, J., Harrick, L., Minnes, P. et al. (1999). Transitions to independent living after ABI. Brain Injury, 13, 311-330.

McColl, M.A., Carlson, P., Johnston, J., Minnes, K., Shue, K., Davies, D. et al. (1998). The definition of community integration: Perspectives of people with brain injuries. Brain Injury, 12 (1), 15-30.

McColl, M.A. (1995). Social support, disability and rehabilitation: A review. Critical Reviews in Physical and Rehabilitation Medicine, 7(4), 315-333.

McColl, M.A., \& Skinner, H.A. (1988). Concepts and measurement of social support in rehabilitation. Canadian Journal of Rehabilitation, 2, 93-107.

Mellick, D., Gerhart, K.A., \& Whiteneck, G.G. (2003). Understanding outcomes based on the post-acute hospitalization pathways followed by persons with traumatic brain injury. Brain Injury, 17(1), 55-71.

Minnes, P., Carlson, P., McColl, M.A., Nolte, M.L., Johnston, J., \& Buell, K. (2003). Community integration: a useful construct , but what does it really mean? Brain Injury, 17, 149-59.

O'Keefe, J. (1994). Long term care and support services for persons with traumatic brain injury. Journal of Head Trauma Rehabilitation, 9(2), 49-60.

O'Reilly, K., \& Pryor, J. (2002). Young people with brain injury in nursing homes: Not the best option. Australian Health Review, 25(3), 46-51.

Petrella, L., McColl, M.A., Krupa, T., \& Johnston, J. (2005). Returning to productive activities: Perspectives of individuals with long-standing brain injuries. Brain Injury, 19(9), 643-655.

Rutterford, N.A., \& Wood, R.L. (2006). Evaluating a theory of stress and adjustment when predicting long-term psychosocial outcome after brain injury. Journal of the International Neuropsychological Society, 12(3), 359-367.

Shavelle, R.M., Strauss, D., Whyte, J., Day, S.M., \& Yu, Y.L. (2001). Long term causes of death after traumatic brain injury. American Journal of Physical Medicine \& Rehabilitation, 80(7), 510-516.

Thurnman, D., Alverson, C., Dunn, K., Guerrero, J., \& Sniezek, J. (1999). Traumatic brain injury in the United States: A public health perspective. Journal of Head Trauma Rehabilitation, 14(5), 602-615.

Tomberg, T., Toomela, A., Pulver, A., \& Tikk, A. (2005). Coping strategies, social support, life orientation and health-related quality of life following traumatic brain injury. Brain Injury, 19(14), 1181-1190. 